\title{
Reliability and validity of D-dimer monitoring for pulmonary thromboembolism in patients with unresectable, advanced or recurrent colorectal cancer treated with bevacizumab
}

\author{
DAN KANEHIRA $^{1,2}$, MASASHI KUSAKABE ${ }^{3}$, SACHIO SHIMIZU $^{1,2}$, JUNICHI SHIMIZU $^{1,2}$, JINSHI IRIKUCHI $^{2}$, \\ AKIHIRO HIRAI $^{4}$, RYOSUKE OKI ${ }^{4}$, TOSHIAKI KATO ${ }^{1,2}$, MASAO YAMASAKI $^{5}$ and KEITA UCHINO ${ }^{1,4}$ \\ Departments of ${ }^{1}$ Chemotherapy, ${ }^{2}$ Pharmacy, ${ }^{3}$ Radiology, ${ }^{4}$ Medical Oncology and ${ }^{5}$ Cardiology, \\ NTT Medical Center Tokyo, Tokyo 141-0022, Japan
}

Received April 26, 2020; Accepted April 29, 2021

DOI: $10.3892 / \mathrm{mco} .2021 .2327$

\begin{abstract}
Pulmonary thromboembolism (PTE) is one of the leading causes of death among cancer outpatients. The aim of the present study was to investigate the reliability and validity of D-dimer monitoring for PTE in patients with unresectable, advanced or recurrent colorectal cancer treated with bevacizumab. A total of 25 patients with advanced colorectal cancer who received bevacizumab combination chemotherapy as primary treatment were retrospectively reviewed. The selection criteria included that D-dimer tests were performed repetitively, and that chest and abdominal contrast-enhanced CT scans were completed. The D-dimer levels and the presence or absence of PTE on CT images were retrospectively examined. Four cases (16\%) were detected as having asymptomatic PTE. The D-dimer values at the onset of PTE were 14.2, 4.6, 1.1 and $0.9 \mu \mathrm{g} / \mathrm{ml}$. The negative predictive value was $90.5 \%$ when $3.0 \mu \mathrm{g} / \mathrm{ml}$ was set as the D-dimer level cutoff value. The incidence of PTE, including asymptomatic PTE, in the present study was higher compared with that reported in previous studies on various types of cancer, of various stages and treated with different chemotherapy regimens. In patients with bevacizumab-treated unresectable, advanced or recurrent colorectal cancer, the D-dimer test was found to be less useful for exclusion diagnosis; however, along with
\end{abstract}

Correspondence to: Mr. Dan Kanehira, Department of Chemotherapy, NTT Medical Center Tokyo, 5-9-22 Higashi-Gotanda, Shinagawa-ku, Tokyo 141-0022, Japan

E-mail: dan.kanehira@gmail.com

Abbreviations: CRC, colorectal cancer; DVT, deep vein thrombosis; NPV, negative predictive value; PTE, pulmonary thromboembolism; VTE, venous thromboembolism

Key words: pulmonary thromboembolism, bevacizumab, D-dimer, negative predictive value, colorectal cancer chest CT, it may be useful in the detection and diagnosis of PTE. However, the determination of the optimal reference values and appropriate measurement timing of D-dimer testing requires further study.

\section{Introduction}

According to the GLOBOCAN 2018 data, colorectal cancer (CRC) is the third leading cause of cancer-related mortality worldwide (1). Despite the high mortality rate of CRC, the substantial progress in multidisciplinary approaches, including chemotherapy, has improved the survival of patients with advanced disease, with a median overall survival of 30 months achieved in clinical trials (2). Overall, the majority of the patients are treated in the outpatient setting.

Pulmonary thromboembolism (PTE) is a serious complication and constitutes one of the leading causes of death among cancer outpatients (3). It is known that tumor-derived tissue factor activates the clotting cascade, and various factors, such as decreased physical activity and mechanical compression of veins by the tumor, may promote thrombus formation in patients with cancer (4). Moreover, bevacizumab, which is used in the treatment of several malignancies, including CRC, is believed to cause vascular endothelial disorders by inhibiting vascular endothelial growth factor and promoting thrombosis (5). An increased risk of venous thromboembolism (VTE), including PTE, has been reported in patients with cancer receiving bevacizumab (6).

The D-dimer test is useful for the exclusion of acute PTE in non-cancer patients; however, as it has high sensitivity but low specificity, it is only useful if the clinical probability is low. Alternatively, although the pre-test probability of PTE is higher among patients with cancer compared with non-cancer patients, the proper utilization of the D-dimer test remains unclear (7).

In patients with unresectable, advanced or recurrent CRC treated with bevacizumab, Mochizuki et al (8) reported that the cutoff value of the D-dimer test for diagnosis of VTE, including PTE, is $3.0 \mu \mathrm{g} / \mathrm{ml}$, with a sensitivity of $75 \%$, specificity of $72 \%$ and negative predictive value (NPV) of $98 \%$, 
and may thus be useful for exclusion diagnosis. Conversely, as cancer progression, as well as thrombosis, increase the $\mathrm{D}$-dimer level, the significance of D-dimer monitoring in the clinical course of patients with cancer remains unclear. Moreover, the recurrence rate of lung metastasis as the site of the first recurrence in patients with CRC is only $5.5 \%$ (9), contrast-enhanced CT scan of the chest is not routine practice in patients undergoing CT scans for routine staging of malignancies, and even contrast-enhanced chest CT scans are associated with high false-negative rates (10). Therefore, the actual association between PTE and D-dimer monitoring remains unknown.

The present study was undertaken to investigate the reliability and validity of D-dimer monitoring in PTE diagnosis by reassessment of CT images in selected patients with unresectable, advanced or recurrent CRC who received bevacizumab as primary treatment and were subjected to contrast-enhanced chest CT examination.

\section{Materials and methods}

Patients and study design. The medical records of 63 patients with histologically confirmed unresectable, advanced or recurrent $\mathrm{CRC}$ who received bevacizumab combination chemotherapy as primary treatment at the NTT Medical Center Tokyo (Tokyo, Japan) between April 2015 and December 2018 were retrospectively reviewed. A total of 25 patients who met the selection criteria [D-dimer tests were performed repetitively, both at baseline and during the bevacizumab treatment period, and abdominal contrast-enhanced chest CT scans performed for any cause (Fig. 1)] were included in the present study. None of the eligible patients had PTE prior to bevacizumab combination chemotherapy or were receiving anticoagulation therapy.

In our selected study population, the presence or absence of PTE and the diagnostic accuracy of D-dimer testing for PTE diagnosis were retrospectively examined, along with an assessment of the patient background and Khorana score (11), which is a score for predicting thrombosis in patients with cancer. The best overall response was assessed according to the Response Evaluation Criteria in Solid Tumors (version 1.1) (12). PTE was retrospectively reassessed by the investigators and a radiologist using chest and abdominal contrast-enhanced CT images.

This study was conducted after obtaining approval from the Institutional Review Board of NTT Medical Center Tokyo (approval no. 19-62).

D-dimer level measurement. A plasma D-dimer latex immunoassay (LIA) was performed using the Nanopia ${ }^{\circledR}$ D-dimer assay kit (Sekisui Medical Co., Ltd.). The measurable D-dimer LIA value ranges from 0.5 to $60 \mu \mathrm{g} / \mathrm{ml}$, while the upper limit of normal is $1.0 \mathrm{mg} / \mathrm{ml}$.

Statistical analysis. Statistical analyses were performed to calculate the sensitivity, specificity and NPV. The cutoff value of the upper limit of the normal D-dimer value was set to $3.0 \mu \mathrm{g} / \mathrm{ml}$ with reference to the report by Mochizuki et al (8). All statistical analyses were performed using EZR software (version 1.35; Saitama Medical Center, Jichi Medical
University, Saitama, Japan). $\mathrm{P}<0.05$ was considered to indicate statistically significant differences.

\section{Results}

Patient characteristics. A total of 25 patients who underwent a total of $250 \mathrm{D}$-dimer tests after receiving bevacizumab combination chemotherapy were included in this investigation. The characteristics of the included patients are summarized in Table I. The Khorana score was 0 points in 22 cases $(88 \%)$, and there were no patients with scores of $\geq 2$ points. The median baseline $\mathrm{D}$-dimer value in our study population was $1.3 \mu \mathrm{g} / \mathrm{ml}$, and the D-dimer value was $>1.0 \mu \mathrm{g} / \mathrm{ml}$, which is the upper limit among patients without cancer, in 15 cases $(60 \%)$. PTE was detected in 4 of the 25 cases $(16 \%)$ on contrast-enhanced CT images, with all cases being asymptomatic.

Clinical course of D-dimer values and characteristics of PTE cases. The clinical course of the D-dimer values and the characteristics of the 4 patients of interest are described in Fig. 2. In Case 1, the baseline D-dimer value was positive at $7.2 \mu \mathrm{g} / \mathrm{ml}$, and at the onset of PTE it further increased to $14.2 \mu \mathrm{g} / \mathrm{ml}$. In Case 2, the baseline D-dimer value was negative at $1.3 \mu \mathrm{g} / \mathrm{ml}$, but at the onset of PTE it was positive at $4.6 \mu \mathrm{g} / \mathrm{ml}$, and the carbohydrate antigen (CA) 19-9 level had also increased from 1,778 to $2,820 \mathrm{U} / \mathrm{ml}$. In Cases 3 and 4, the baseline D-dimer values were negative and were found to have further decreased at the onset of PTE, whereas the CA19-9 and carcinoembryonic antigen levels had also decreased. PTE in Case 1 was asymptomatic; however, increased D-dimer levels were detected on blood tests. Therefore, contrast-enhanced CT scan was performed for suspected PTE, and PTE was detected. PTE in Cases 2, 3 and 4 was detected by reassessment of contrast-enhanced CT images.

The sensitivity, specificity and NPV were 50.0, 90.5 and $90.5 \%$, respectively, when $3.0 \mu \mathrm{g} / \mathrm{ml}$ was set as the D-dimer cutoff value (Table II).

\section{Discussion}

Despite its retrospective design and small sample size, to the best of our knowledge, this is the first study to evaluate the reliability and validity of D-dimer monitoring for PTE limited to patients with unresectable, advanced or recurrent CRC treated with bevacizumab as primary treatment. CRC is a type of cancer not included in the Khorana score, and it is often treated by bevacizumab in the primary to tertiary treatment setting. Therefore, CRC was selected as the best target to investigate PTE in the present study.

PTE in patients with cancer is associated with high recurrence rate and poor prognosis, despite it being asymptomatic or an incidental finding. Therefore, it is important to accurately diagnose even asymptomatic PTE, and the same treatment is recommended for symptomatic and asymptomatic patients, including patients diagnosed incidentally (13). The PTE cases detected in the present study included asymptomatic PTE that was incidentally diagnosed upon reassessment of contrast-enhanced chest CT images for routine staging of malignancy, indicating that it is difficult to detect all cases in 
Table I. Patient characteristics and incidence rate of PTE.

\begin{tabular}{|c|c|}
\hline Characteristics & No. $(\%)$ \\
\hline Age, years (range) & $66(42-81)$ \\
\hline \multicolumn{2}{|l|}{ Sex } \\
\hline Male & $12(48.0)$ \\
\hline Female & $13(52.0)$ \\
\hline \multicolumn{2}{|l|}{ Concurrent regimen } \\
\hline mFOLFOX6 & $19(76.0)$ \\
\hline CapeOX & $3(12.0)$ \\
\hline FOLFIRI & $2(8.0)$ \\
\hline CPT-11 & $1(4.0)$ \\
\hline \multicolumn{2}{|l|}{ Best overall response $^{\mathrm{a}}$} \\
\hline Complete response & $0(0)$ \\
\hline Partial response & $7(28.0)$ \\
\hline Stable disease & $14(56.0)$ \\
\hline Progressive disease & $4(16.0)$ \\
\hline \multicolumn{2}{|l|}{ Khorana score } \\
\hline 0 & $22(88.0)$ \\
\hline 1 & $3(12.0)$ \\
\hline$\geq 2$ & $0(0)$ \\
\hline Baseline D-dimer, $\mu \mathrm{g} / \mathrm{ml}$ (range) & $1.3(0.4-16.9)$ \\
\hline $\begin{array}{l}\text { Baseline carcinoembryonic antigen, } \\
\mathrm{ng} / \mathrm{ml} \text { (range) }\end{array}$ & $22.9(1.7-2,330.0)$ \\
\hline $\begin{array}{l}\text { Baseline carbohydrate antigen } 19-9 \text {, } \\
\mathrm{U} / \mathrm{ml} \text { (range) }\end{array}$ & $120(0-284,810)$ \\
\hline Number of bevacizumab cycles (range) & $10(2-36)$ \\
\hline $\begin{array}{l}\text { Number of D-dimer tests per } \\
\text { patient (range) }\end{array}$ & $8(1-33)$ \\
\hline $\begin{array}{l}\text { Number of enhanced CT scans per } \\
\text { patient (range) }\end{array}$ & $3(1-7)$ \\
\hline PTE cases & $4(16.0)$ \\
\hline
\end{tabular}

${ }^{a}$ RECIST criteria, version 1.1. Results are presented as the median (range) or number (\%). mFOLFOX6, oxaliplatin, L-leucovorin and 5-FU; CapeOX, capecitabine and oxaliplatin; FOLFIRI, irinotecan and L-leucovorin. 5-FU; 5-FU, 5-fluorouracil; PTE, pulmonary thromboembolism.

clinical practice. Therefore, the primary purpose was to investigate whether blood tests, such as the D-dimer testing, would be useful for complementing PTE diagnosis.

However, the high NPV, which is a characteristic of the $\mathrm{D}$-dimer test in non-cancer patients, may be less reliable in patients with cancer due to the higher incidence of PTE compared with non-cancer patients. Furthermore, despite the fact that the D-dimer test is a sensitive method for the diagnosis of acute PTE in non-cancer patients, the sensitivity of D-dimer test was only $50 \%$ in patients with cancer, with a cutoff value of $3.0 \mu \mathrm{g} / \mathrm{ml}$. These results suggest that periodic chest CT scans are necessary, even if the D-dimer test is negative. In other words, the D-dimer test has low sensitivity in patients with bevacizumab-treated unresectable, advanced or recurrent $\mathrm{CRC}$, and may only be useful in the detection and diagnosis of PTE, rather than the exclusion of PTE. In
Table II. Diagnostic accuracy of D-dimer test for PTE.

\begin{tabular}{llr}
\hline & \multicolumn{2}{c}{$\begin{array}{c}\text { Number of patients } \\
(\mathrm{n}=25)\end{array}$} \\
\cline { 2 - 3 } Variables & PTE (+) & PTE (-) \\
\hline D-dimer level, $\mu \mathrm{g} / \mathrm{ml}$ & & 2 \\
$>3.0$ & 2 & 19 \\
$\leq 3.0$ & 2 & \\
Measure of accuracy, \% & & 50.0 \\
Sensitivity & & 90.5 \\
Specificity & & 50.0 \\
Positive predictive value & & 90.5 \\
Negative predictive value & & \\
\hline
\end{tabular}

PTE, pulmonary thromboembolism.

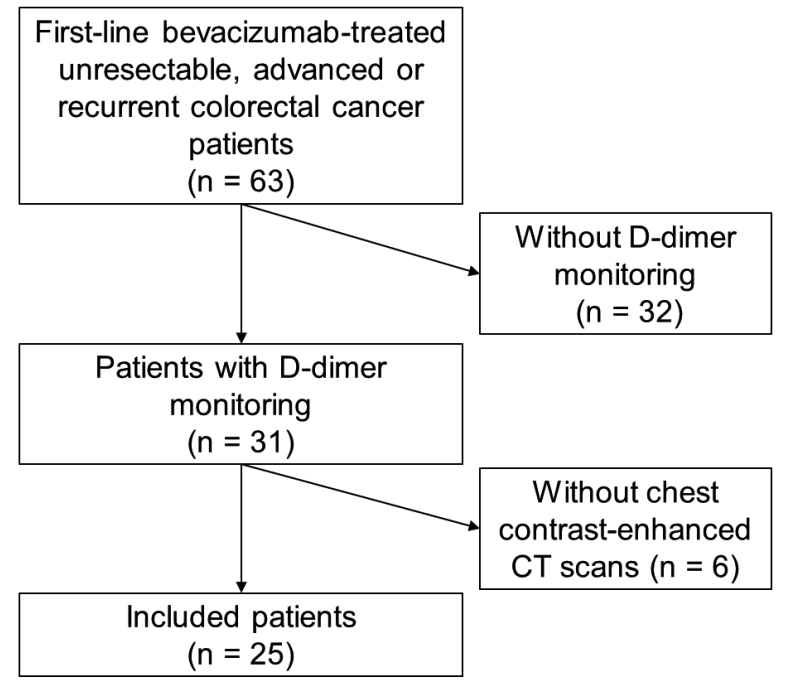

Figure 1. Flowchart of patient selection. Inclusion criteria were patients with histologically confirmed unresectable, advanced or recurrent colorectal cancer who received bevacizumab combination chemotherapy as a primary treatment. Patients were excluded if they had not been subjected to D-dimer monitoring or contrast-enhanced chest CT scan.

addition, patients with cancer have additional factors that may also affect D-dimer levels, such as age, inflammation and tumor progression, and the D-dimer levels may decrease with disease control by treatment; therefore, D-dimer level routine monitoring may be important, in addition to its measurement at baseline and at the timing of appearance of suspicious symptoms. The cutoff value of the D-dimer test in PTE diagnosis requires further verification, but this could not be performed in the present study owing to the insufficient sample size. However, if D-dimer levels rapidly increase during routine monitoring, as in Cases 1 and 2 reported herein, the possibility of PTE should be considered.

There were several major limitations to the present study. First, this was a retrospective observational study with a small sample size. Second, PTE was evaluated by 


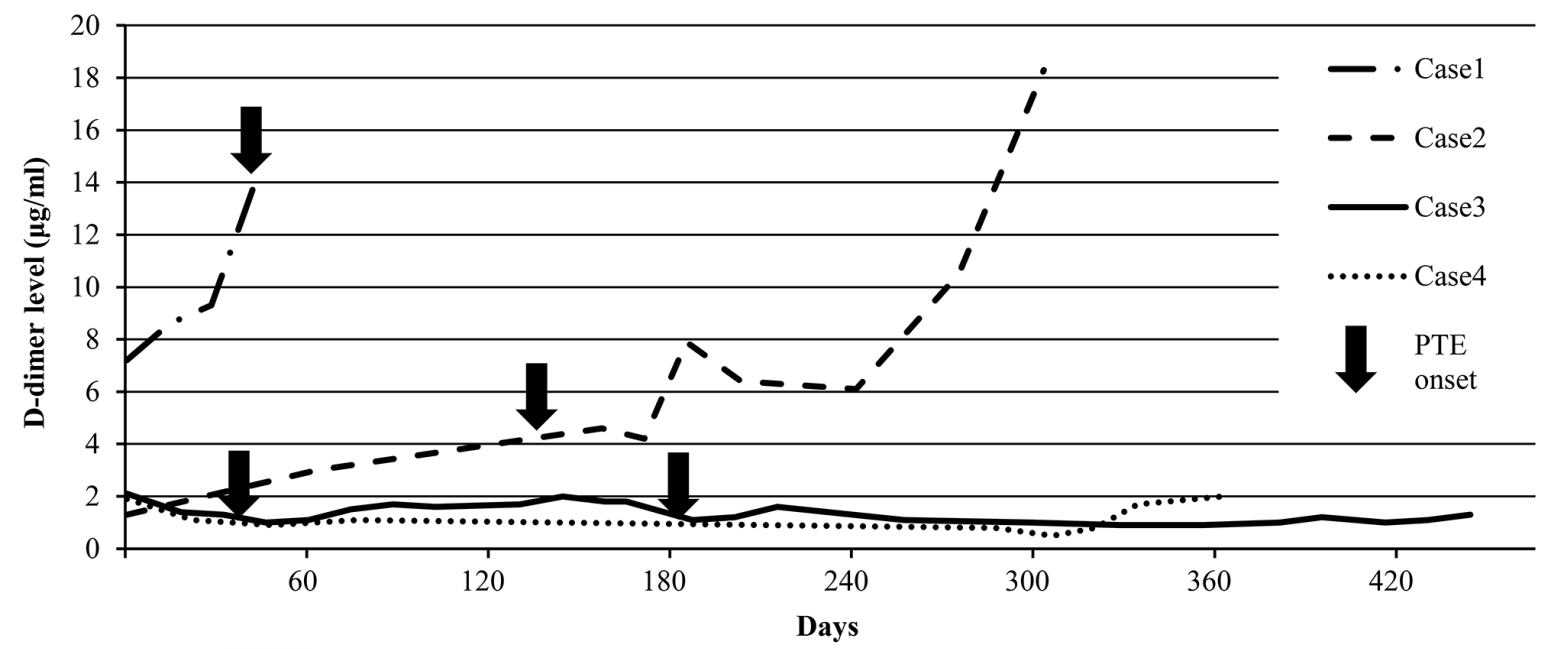

\begin{tabular}{cccccccccccc} 
Case & $\begin{array}{c}\text { Age } \\
\text { (years) }\end{array}$ & Sex & $\begin{array}{c}\text { Concurrent } \\
\text { regimen }\end{array}$ & $\begin{array}{c}\text { Khorana } \\
\text { score }\end{array}$ & $\begin{array}{c}\text { Days after } \\
\text { 1st bevacizumab } \\
\text { given (days) }\end{array}$ & \multicolumn{2}{c}{ D-dimer $(\mu \mathrm{g} / \mathrm{ml})$} & \multicolumn{2}{c}{ CEA $(\mathrm{ng} / \mathrm{ml})$} & \multicolumn{2}{c}{ CA19-9 $(\mathrm{U} / \mathrm{ml})$} \\
\hline 1 & 68 & Female & mFOLFOX6 & 0 & 41 & 7.2 & 14.2 & 91.7 & 34.8 & 1722 & 934 \\
2 & 52 & Male & mFOLFOX6 & 0 & 136 & 1.3 & 4.6 & 72.9 & 53.7 & 1778 & 2820 \\
3 & 73 & Female & mFOLFOX6 & 0 & 182 & 2.1 & 1.1 & 53.0 & 15.0 & 331 & 23 \\
4 & 68 & Female & mFOLFOX6 & 1 & 37 & 1.9 & 0.9 & 13.7 & 12.1 & 252 & 128
\end{tabular}

Figure 2. Clinical course of the D-dimer values and characteristics of the four PTE cases. Arrows indicate PTE onset. The D-dimer values were increased at the onset of PTE in Cases 1 and 2, but baseline D-dimer values were negative and further decreased along with tumor markers in Cases 3 and 4 . PTE, pulmonary thromboembolism; CA19-9, carbohydrate antigen 19-9; CEA, carcinoembryonic antigen.

contrast-enhanced chest CT images, but without considering the possibility of deep vein thrombosis (DVT) in the lower limbs or in the injection site of the central venous catheter. There may be an association between PTE and DVT, and the source of PTE may be thrombi formed in the veins in the lower limbs or pelvis. In particular, the lower limbs are rarely evaluated by CT scans for the purpose of disease assessment in patients with CRC, and ultrasonography is generally only performed when suspicious symptoms are observed. The PTE cases in the present study may have developed DVT prior to the onset of PTE, and the relevance between the onset of DVT and the variation of D-dimer levels and NPV was not elucidated in this study. Finally, on routine monitoring of D-dimer levels, the reasons for the low sensitivity of D-dimer testing may include the negative conversion of the D-dimer levels due to the organization of the thrombus with the lapse of time after thrombus formation and the detection of minute thromboembolism by reassessment of contrast-enhanced CT scans. Future research should be aimed at elucidating this issue.

Despite these limitations, it was herein demonstrated that PTE, including asymptomatic and incidentally diagnosed cases, appears to be a frequent occurrence among patients with CRC, such as those included in the present study, and the D-dimer test may be less useful for exclusion diagnosis, but may prove useful for appropriate PTE detection and diagnosis in addition to chest CT scans. In addition, D-dimer level monitoring is hypothesized to be important not only at baseline, but also during treatment. However, the optimal reference values and the appropriate measurement and timing of D-dimer testing require further study.

\section{Acknowledgements}

The authors would like to thank the staff of Chemotherapy Center, NTT Medical Center Tokyo.

\section{Funding}

No funding was received.

\section{Availability of data and materials}

All data generated or analyzed during the present study are included in this published article.

\section{Authors' contributions}

DK and KU: Study conception and design. DK, MK and KU: Data collection and analysis. DK, MK and KU have seen and can confirm the authenticity of the raw data. DK and KU: Drafting of the manuscript. SS, JS, JI, AH, RO, TK and MY: Critical review and revision of the manuscript for important intellectual content. All the authors contributed to the discussion and have read and approved the final manuscript.

\section{Ethics approval and consent to participate}

The present study was approved by the Ethics Committee of NTT Medical Center Tokyo (approval no. 19-62). As this was a retrospective observational study, consent was not obtained from individual patients. An information disclosure 
document about this study was created and published for the study patient, guaranteeing the opportunity for the study patient to refuse.

\section{Patient consent for publication}

Not applicable.

\section{Competing interests}

The authors declare that they have no competing interests.

\section{References}

1. Bray F, Ferlay J, Soerjomataram I, Siegel RL, Torre LA and Jemal A: Global cancer statistics 2018: GLOBOCAN estimates of incidence and mortality worldwide for 36 cancers in 185 countries. CA Cancer J Clin 68: 394-424, 2018.

2. Cremolini C, Loupakis F, Antoniotti C, Lupi C, Sensi E, Lonardi S, Mezi S, Tomasello G, Ronzoni M, Zaniboni A, et al FOLFOXIRI plus bevacizumab versus FOLFIRI plus bevacizumab as first-line treatment of patients with metastatic colorectal cancer: Updated overall survival and molecular subgroup analyses of the open-label, phase 3 TRIBE study. Lancet Oncol 16: 1306-1315, 2015.

3. Khorana AA: Venous thromboembolism and prognosis in cancer. Thromb Res 125: 490-493, 2010.

4. Fernandes CJ, Morinaga LTK, Alves JL, Castro MA, Calderaro D, Jardim CVP and Souza R: Cancer-associated thrombosis: The when, how and why. Eur Respir Rev 28: 180119, 2019.

5. Nalluri SR, Chu D, Keresztes R, Zhu X and Wu S: Risk of venous thromboembolism with the angiogenesis inhibitor bevacizumab in cancer patients: A meta-analysis. JAMA 300: 2277-2285, 2008.

6. Alahmari AK, Almalki ZS, Alahmari AK and Guo JJ Thromboembolic events associated with bevacizumab plus chemotherapy for patients with colorectal cancer: A meta-analysis of randomized controlled trials. Am Heal Drug Benefits 9: 221-231, 2016.
7. Crawford F, Andras A, Welch K, Sheares K, Keeling D and Chappell FM: D-dimer test for excluding the diagnosis of pulmonary embolism. Cochrane Database Syst Rev 2016: CD010864, 2016.

8. Mochizuki S, Yoshino T, Kojima T, Fuse N, Ikematsu H, Minashi K, Yano T, Tahara M, Kaneko K, Doi T, et al: Therapeutic significance of a D-dimer cut-off level of $>3 \mu \mathrm{g} / \mathrm{ml}$ in colorectal cancer patients treated with standard chemotherapy plus bevacizumab. Jpn J Clin Oncol 40: 933-937, 2010.

9. Hashiguchi Y, Muro K, Saito Y, Ito Y, Ajioka Y, Hamaguchi T, Hasegawa K, Hotta K, Ishida $\mathrm{H}$, Ishiguro M, et al: Japanese society for cancer of the colon and rectum (JSCCR) guidelines 2019 for the treatment of colorectal cancer. Int J Clin Oncol 25: $1-42,2020$.

10. van Es N, Bleker SM and Di Nisio M: Cancer-associated unsuspected pulmonary embolism. Thromb Res 133 (Suppl 1): S172-S178, 2014

11. Khorana AA, Kuderer NM, Culakova E, Lyman GH and Francis CW: Development and validation of a predictive model for chemotherapy-associated thrombosis. Blood 111: 4902-4907, 2008.

12. Eisenhauer EA, Therasse P, Bogaerts J, Schwartz LH, Sargent D, Ford R, Dancey J, Arbuck S, Gwyther S, Mooney M, et al: New response evaluation criteria in solid tumours: Revised RECIST guideline (version 1.1). Eur J Cancer 45: 228-247, 2009.

13. Key NS, Khorana AA, Kuderer NM, Bohlke K, Lee AYY, Arcelus JI, Wong SL, Balaban EP, Flowers CR, Francis CW, et al: Venous thromboembolism prophylaxis and treatment in patients with cancer: ASCO clinical practice guideline update. J Clin Oncol 38: 496-520, 2020. 